\title{
The Research on Lung Cancer Significant Detection Combined with Shape Feature of Target
}

\author{
Guilai Han, Yuan Jiao and Xiaoling Li \\ Faculty of Information Technology Hainan Medical College Haikou, China
}

\begin{abstract}
At present, the research on detection of lung cancer includes the sample image segmentation, extracting visual features of lung cancer and generating the classification model by training learning, then according to the classification model generated to classify the inspected images. But this kind of method usually needs a large amount of calculation and speed is slow. In order to find the region of interest as soon as possible and improve the detection speed, this paper attempts to introduce the current popular Itti visual attention model into the lung cancer detection. However, because medical images usually have low contrast, the Itti method is not directly applied to extract the region of interest in medical image. Therefore the selective visual attention mechanism combined with shape feature of target is proposed. Firstly some primary features are chosen, such as gray scale, direction, corner point and edge to generate saliency map, and then the significant regions are segmented and judged. Compared to popular lung cancer detection method, this method can improve the detection rate of suspected lung cancer and has great significance for the early detection, early diagnosis and treatment of lung cancer.
\end{abstract}

\section{Introduction}

Malignancies which are commonly known as cancers bring great harm to patients and their families and consume too much valuable social medical resources. But the effect of treatment is very little. Lung cancer is one of the largest cancers which hazards human life and health. The mortality rate of the lung cancer is much higher than other cancers, and is increasing year by year. The statistics shows that lung cancer has replaced liver cancer to become the highest death rate of cancer, accounting for $22.7 \%$ of all cancer deaths, and the incidence and mortality rates continue to rise rapidly. In 2014, the incidence rate of lung cancer in China grew $26.9 \%$.If we do not take effective measures, Chinese lung cancer patients will reach 100 million in 2025, and China will become the world's first lung cancer country. At average, one person died from lung cancer every 30 seconds in the world. In fact Lung cancer has been the first deputy killer. Early detection and early diagnosis is an important measure to reduce lung cancer mortality. If lung cancer can be diagnosed and treated at an early stage, the 5-year survival rate will increase from $14 \%$ to $49 \%$ [1].

The important feature of lung cancer early is pulmonary nodules, especially malignant pulmonary nodules have burr and leaf features. Using medical image, doctors can find cancer in time, and provide a Diagnosis opinion. Computed tomography (CT) is the most common chest radiographic image which is widely used in lung tumors and lung nodules detection. Due to the huge number of images, the analysis of staff chest CT image is a boring and tedious process. Especially for a high-resolution scan image, a number of images scanned only a patient can reach tens or even hundreds of layers, which bring the enormous workload to doctors, thereby increase the chances of misdiagnosis and missed diagnosis. Computer-aided detection (CAD) for CT images can be automatically prompted to perform the analysis of the image of staff suspicious pulmonary nodules. It can effectively improve diagnostic accuracy and reduce the rate of misdiagnosis. Using computeraided detection to detect a variety of early cancers has become a focus of attention.

\section{Computer-aided Detection and Visual Attention Mechanisms}

The current research on computer-aided detection of early lung cancer includes the sample image segmentation, extracting visual features of lung cancer, generating the classification model by training and learning, and according to the classification model to classify the inspected images. However, this kind of method usually needs a large amount of calculation and the speed is slow which severely restricted the use of such software in the actual clinic. In order to find the region of interest as soon as possible and improve the detection speed, the visual attention model is introduced into lung cancer detection.

Human can quickly search for the region of interest from complex scenes. The ability to choose is called visual attention mechanism. The role of visual attention 
mechanism lies in extracting visual information, sorting things by priority, then dealing with higher priority information and ignoring lower priority information at the fastest speed. The traditional image processing technology processes all of the pixels unitary, regardless of priority. But in many image analysis tasks, such as image retrieval, scene analysis, target identification and target tracking, they have priorities. So the regardless of priority image processing not only wastes computing resources, but also increases the complexity of the analysis process. So the study of visual attention mechanisms has more important scientific value and practical significance.

Now that there is not yet a unified mechanism of theoretical and computational model of visual attention, the visual attention model Itti et al [2] proposed is more recognized academic, which using Gaussian pyramid and considering three visual features of brightness, color, direction to obtain multi-scale significant measure by Center-surround operator, and the final saliency map obtained through fusion and normalization, finally using saliency map to evaluate a given area differences in the surrounding area.

The Itti visual attention model has achieved good effects in the natural image target detection, but the effect is not ideal when it is used in medical images which are mainly gray images. Especially in the early stages of lung cancer, the focus on lung cancer is not the most significant region in medical images, and sometimes it is very difficult to be found. Although Itti visual attention model can obtain the position of a significant target, it does not reflect the size and shape of significant goal. For this reason, the selective visual attention mechanism combined with the shape features of lung cancer, was proposed to improve Itti visual attention model in this paper. The specific method extracts the features of gray, directions, corners, edges and local entropy to generate feature saliency map.

\section{Lung Cancer Significant Detection Combined with Shape Feature of Target}

Because lung cancer are usually small in the whole chest CT images, the Itti method is used to detect the most significant area which is not the area of lung cancer in most cases. So this paper attempts to combine the shape features of lung cancer to detect saliency. Lung cancer usually has features of burr and leaf. In order to simplify calculations, the primary visual features which are simple and easy to be got, such as gray, direction, corner, edge and local entropy, are used to generate saliency map of specific target area. The flow diagram of visual attention model combined with shape feature of target was shown in Fig. 1.

Human visual system based on different types of receptive field to extract the image information step by step. In recent years, neuroscientists have acquired a lot of experimental results about the characteristics and types of receptive field through continuous research. In 1965, Rodieck pointed out that the receptive field of human eye can be expressed by two Gaussian functions difference
[3]. The receptive field was expressed by polar coordinates as eq. 1 .

$$
D O G(r)=A \exp \left(-r^{2} / \sigma_{A}^{2}\right)-B \exp \left(-r^{2} / \sigma_{B}^{2}\right)
$$

Among them, $r$ expresses the distance between any point and the central point in receptive field.

Neurobiology found through further study, the difference of Gaussian function (DOG) and center surrounding receptive field are very similar. Due to the difference between receptive field and DOG function is very small, DOG filter is usually adopted to simulate the human eye receptive fields in the visual design attention mechanism mode [4] 1. In the visual attention mechanism model, the two concepts of multi scale and centersurround difference are put forward based on receptive field.

In order to obtain multi scale sampling, the three-tier pyramid model is adopted in fig. 1 . The original image is the first layer. The second layer and the third layer are sequentially sampled on the basis of the upper layer, and their size is $1 / 2$ of the upper layer. The different features of the image were filtered by using center-surround operation. By comparing the differences between the candidate region and the surrounding area in the brightness, direction, corner, edge and local entropy of the underlying features, the saliency maps of respective features were obtained.

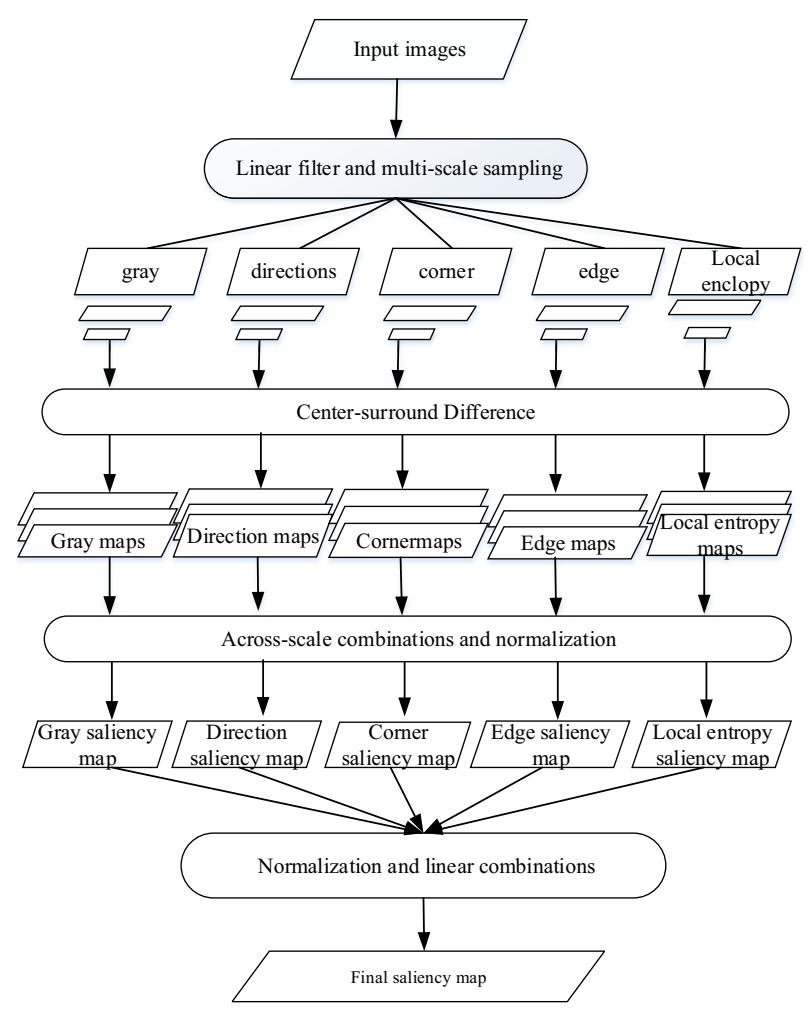

Figure 1. The flow diagram of visual attention model combined with shape feature of target

In the multi feature map integration stage, the feature maps which coming from the same feature and different layers were integrated by across scale combination and 
normalization. For the saliency maps of different feature $\mathrm{s}$, according to the feature integration theory, different feature information of significant measure is not the same, so it is necessary to integrate the different feature channels to obtain the final saliency map by normalization and linear or nonlinear method.

In order to get better results, median filter was used firstly to eliminate the influence of noise, and the lung parenchyma image shown in Fig. 2 was got from chest CT image through image segmentation.

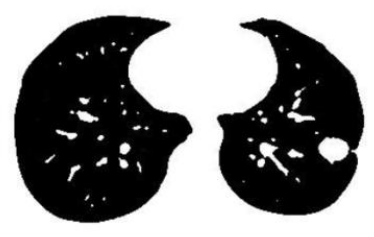

Figure 2. Lung parenchyma image

\subsection{Gray feature}

Gray is one of the most important features of medical image, and it is the most direct expression of the difference between the lesion and normal human body. After a series of operations, such as multi scale sampling, center-surround difference and normalization can get gray saliency map, as shown in fig. 3 .

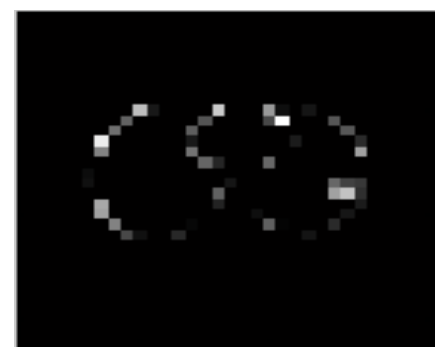

Figure 3. Gray feature saliency map

\subsection{Direction}

Using Gabor filter on the brightness image to filter in the four direction of $0^{\circ}, 45^{\circ}, 90^{\circ}, 135^{\circ}$, to acquire the direction images. After multi scale sampling, centersurround difference and normalization can get direction saliency map, as shown in fig.4.

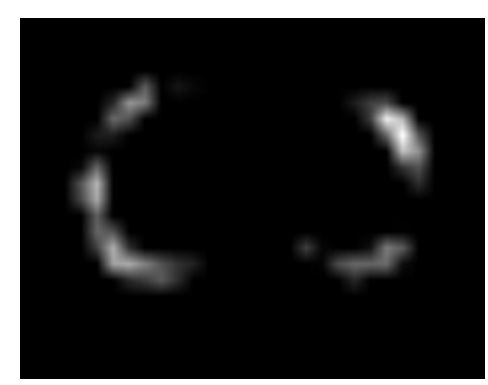

Figure 4. Direction feature saliency map

\subsection{Corner}

Corner is the point where the image brightness changes sharply or the edge of the image has maximum curvature. While retaining the important characteristics of image, the corner can reduce the amount of information and improve the calculation speed effectively. The identification of corner points is usually used in a local small window to slide in the image, through the observation of the gray value changes in the window to achieve. Harris corner detection operator and SUSAN detection operator are used commonly corner detection operators[5].Harris corner operator is chosen as an example in this paper. Harris corner feature and its saliency map are shown in Fig. 5.

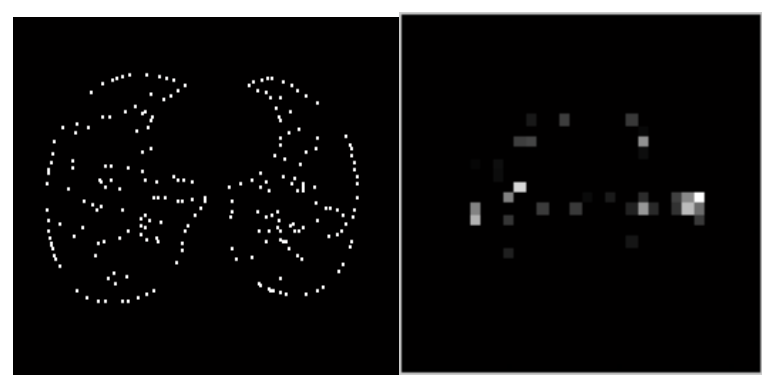

Figure 5. Harris corner feature and its saliency map

\subsection{Edge}

An edge is a collection of pixels whose gray value changes sharply. As the most important basis of image segmentation, edge often exists between target and background region. Edge is the sign of the location and it is not sensitive to the change of the gray value. Therefore, the edge is an important feature of image matching. sobel operator, log operator, canny operator and so on are Commonly used edge detection operators. Canny operator which has the most excellent performance in all of the operators is widely used by everyone [6].Canny edge feature and its saliency map are shown in Fig.6.
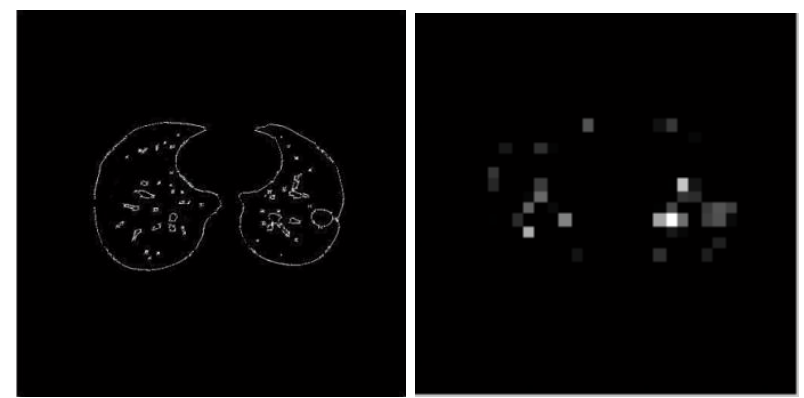

Figure 6. Canny edge feature and its saliency map

\subsection{Entropy}

Entropy is the concept used to measure the amount of information in information theory. If the system is more orderly, the information entropy is lower; on the contrary, if the system is more chaotic, the information entropy is higher. By the concept of entropy in information theory, the entropy of the image is a measure of the amount of 
information contained in the image. The bigger the entropy of the image is, the more the image information is. Then the region is more likely to be the region of interest (ROI)in the image [7].

Assume that the probability of the pixel whose gray level is $m$ appears as in the image. According to the definition of the information entropy, the information entropy of the gray level can be defined as eq. 2 .

Where in For the gray image, the information entropy of the image can be defined as eq. 3 .

It can be seen from the definition of the entropy of the image that the image information entropy only considers the global statistics of the image, and the spatial distribution information of the image is discarded. Therefore, in order to represent local features of the image, the concept of local entropy is introduced, whose idea is that the image is divided into a number of the same size sub images, and then the entropy of each sub image is calculated. Local entropy feature and its saliency map are shown in Fig.7.
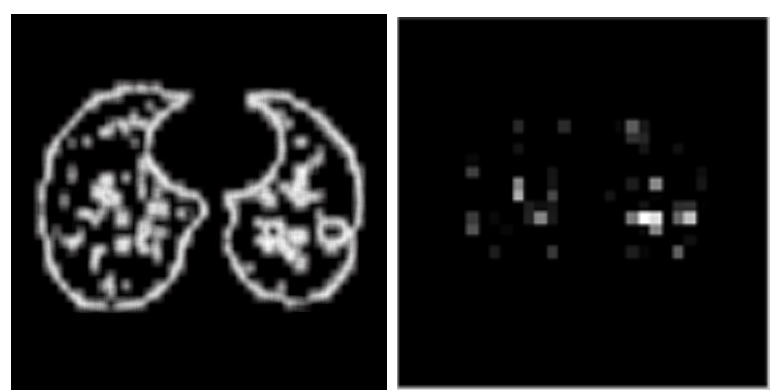

Figure 7. Local entropy feature and its saliency map

\section{Conclusion}

Because the medical image is usually a gray image, its color features can't be got, The saliency map shown in Fig.8 is obtained by the two features of brightness and direction based on Itti visual attention mechanism. The final saliency map shown in Fig.9 was acquired by the visual attention model combined with shape feature of target. Fig.8 and Fig.9 can be seen in contrast, the saliency map obtained by combining with the shape features of lung cancer can better highlight the region of interest which the lung cancer corresponds to than Itti feature saliency map.

In order to more quickly find lung cancer region, some primary visual features such as corners, edges and local entropy are introduced to improve the detection of lung cancer in this paper. Experiments show that the method can be faster and more accurate to find significant area compared to the Itti method. Using vision attention mechanism to find the significant region is only rough location. In order to more accurately detect lung cancer and decrease the false positive rate, segmenting the significant areas in saliency map is needed. And it is need to extract more detailed features and to classify according to classification model established on the basis of sample image.

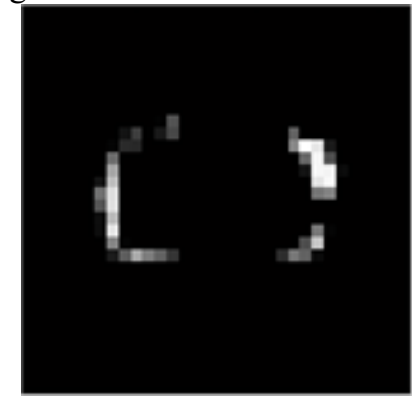

Figure 8. Itti feature saliency map

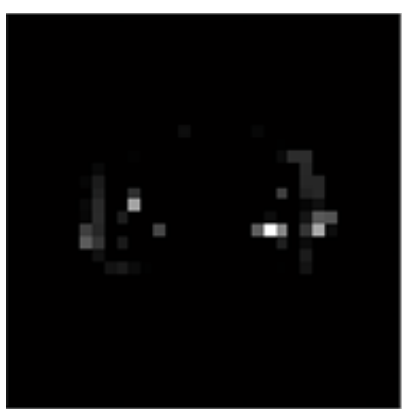

Figure 9. Final saliency map

\section{Acknowledgment}

This research was supported by the Hainan Province Nature Science Fund Item (Grant No:20156224).

\section{References}

1. H. Jie, C. H. Wanqing, Annual report on status of cancer in China, 2014, (TsingHua University Pres, 2014)

2. L. Itti, C. Koch , E. Niebur, A model of saliencybased visual attention for rapid scene analysis, TPAMI, 20,6(1998).

3. Rodieck R W, Stone J J. Analysis of receptive fields of cat retina ganglion cells. JON, 28,17(1965)

4. Z. Ming, L. Youfu, M. Qinghao, Y. Ting , L. Jian, H. Tiemao, Finding Regions of Interest Based on Scale-Space Keypoint Detection. CCIS, 202, 8(2011).

5. ZH. Congpeng, W. Xueguang, Rectangle detection based on Harris corner. OPE, 89, 7(2014).

6. T. Lu-lu, ZH. Qi-can, H. Song, An Improved Algorithm for Canny Edge Detection with Adaptive Threshold. OEE, 38, 6 (2011).

7. SH. Yuhao, H. Weiwang, SH. Rui, Y. Yangchao, O. Congjie, Novel image segmentation algorithm based on entropy and clustering theory, ARC, 33,6 (2016). 